\title{
TRATO DIGNO DE ENFERMERÍA DURANTE 2010-2015 EN EL SISTEMA NACIONAL DE INDICADORES DE CALIDAD
}

\author{
NURSING DIGNIFIED TREATMENT DURING 2010-2015 IN THE QUALITY INDICATORS NATIONAL SYSTEM
}

SILVIA GUADALUPE SALMÓN VEGA*

MARCELA PADILLA LANGURÉ**

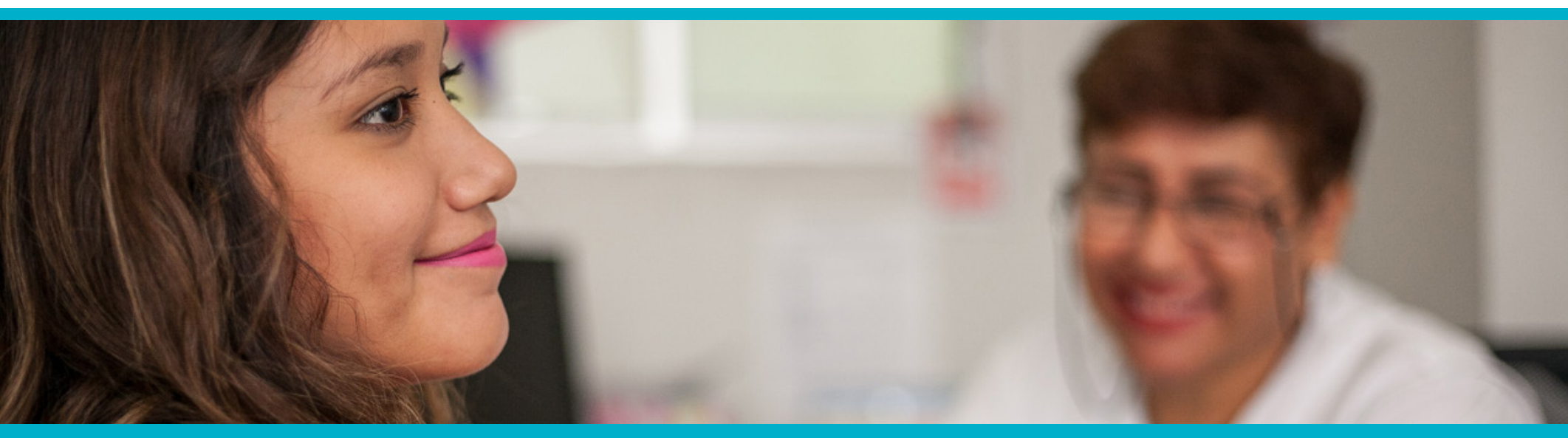

\section{ABSTRACT}

In this work the behavior of the indicatodignified treatment nursing occurs during the period 2010 to 2015 in the Health Quality Indicators National System, with the aim of describing the perception of quality of care received by users health services and identify this indicator variables representing a major challenge for the nurse. The information analyzed was obtained from the Web site of the General Direction Quality and Health Education. The results indicate that during the period 2010-2011 was not met with the standard minimum score of $95 \%$ expected by meeting the criteria established for this indicator. The limiting criteria were percentage of patients to whom the nurse (o): a) greets him in a friendly, b) is presented, and c) is directed by name. From 2012 to 2015 it met the standard, which is suggestive that the measurement of this indicator has detected areas of opportunity and implement continuous improvement strategies, on the other hand also indicates the ethical and humanistic approach to care that characterizes nursing profession.

Keywords: Quality indicators, nursing, healt nursing care.

\section{RESUMEN:}

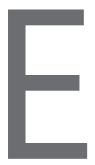
n este trabajo se presenta el comportamiento del indicador de trato digno de enfermería durante el periodo 2010 a 2015 en el Sistema Nacional de Indicadores de Calidad en Salud, con el objetivo de describir la percepción de la calidad de la atención recibida por los usuarios de los servicios de salud, así como identificar las variables de este indicador que representen un mayor reto para el profesional de enfermería. La información analizada se obtuvo del portal de internet de la Dirección General de Calidad y Educación en Salud. Los resultados indican que durante el periodo 20102011 no se cumplió con el estándar de calificación mínima de $95 \%$ esperada por el cumplimiento de los criterios establecidos para este indicador. Los criterios limitantes fueron porcentaje de pacientes a los cuales la enfermera (o): a) lo saluda en forma amable, b) se presenta, y c) se dirige por su nombre. De 2012 a 2015 se cumplió con el estándar, lo cual sugiere que la medición de este indicador ha permitido detectar áreas de oportunidad e implementar estrategias de mejora continua, por otro lado también indica el enfoque ético y humanístico en la atención que caracteriza la profesión de enfermería.

\footnotetext{
*Dra. en Ciencia de Materiales, Maestra en Gestión de Salud Institucional, Licenciada en Enfermería y Obstetricia, Profesor de asignatura, Departamento de Enfermería, Universidad de Sonora. Hospital General "Dr. Fernando Ocaranza", ISSSTE, Hermosillo, Sonora. Correo electrónico: silviasalmon@ yahoo.com.mx

** Maestra en Ciencias de Enfermería. Docente de tiempo completo. Departamento de

\section{Palabras claves:}

Indicadores de calidad, enfermería, atención de enfermería (DeCS, BIREME). 


\section{INTRODUCCIÓN}

La Organización Mundial de la Salud define calidad de asistencia como el proceso en que cada enfermo recibe un conjunto de servicios diagnósticos y terapéuticos, en que teniendo en cuenta todos los factores del paciente y del servicio médico, se logra obtener el mejor resultado con el mínimo riesgo de efectos iatrogénicos y la máxima satisfacción del paciente ${ }^{(1)}$.

En este contexto, Avedis-Donabedian ${ }^{(2-3)}$ propuso el componente técnico y el componente interpersonal como elementos fundamentales de la calidad en la atención de salud susceptibles de generar satisfacción. El componente técnico, es empleo de la ciencia y tecnología en beneficio de la salud, mientras que el componente interpersonal es el aspecto humanitario de la relación entre el paciente y el personal de salud.

Sin embargo, la calidad de atención en salud es un reto permanente a nivel mundial que requiere involucramiento de los gobiernos, profesionales de salud y de la población en su conjunto, pues incluye recursos, políticas públicas, actitudes y voluntades.

En nuestro país, con la convicción de mejorar los servicios de salud y situar la calidad en la agenda permanente, el Gobierno Federal a través de la Dirección General de Calidad y Educación en Salud (DGCES) implemento el Sistema Nacional de Indicadores de Calidad en Salud (INDICAS) como una estrategia que permite registrar y monitorear indicadores de calidad en establecimientos de atención médica públicos y privados ${ }^{(4-5)}$.

Dentro de este sistema, la calidad de los servicios de enfermería se concibe como la atención oportuna, personalizada, humanizada, continua y eficiente que brinda el personal de enfermería, de acuerdo con estándares definidos para una práctica profesional competente y responsable, con el propósito de lograr la satisfacción del usuario y del prestador de servicios de enfermería. ${ }^{(6-9)}$ Por tanto, la atención de enfermería está sujeta a evaluación mediante indicadores de calidad en enfermería, los cuales se consideran en el índice de enfermería en segundo nivel, dividido en 6 indicadores que a su vez están constituidos por 46 criterios o variables ${ }^{(10)}$.

El registro y monitoreo de los indicadores de enfermería en el Sistema Nacional de Salud mediante la estrategia INDICAS tiene su origen en 2004, con la medición de tres indicadores. El indicador de ministración de medicamentos vía oral, vigilancia y control de venoclisis, y trato digno de enfermería. (6) Posteriormente, se incorporaron los indicadores de prevención de infección en vías urinarias, prevención de caídas a pacientes y prevención de úlceras por presión a pacientes hospitalizados.
Actualmente, a través de estos seis indicadores es posible evaluar el componente técnico e interpersonal del cuidado de enfermería en el segundo nivel de atención de salud. Sin embargo, al ser considerada enfermería como la ciencia de cuidado, el trato digno representa el componente interpersonal esencial para proveer cuidado humanizado en la relación personapersona ${ }^{(8-9)}$.

Operacionalmente, el trato digno de enfermería se define como la percepción que tiene el paciente o familiar del trato y la atención proporcionada por el personal de enfermería durante su estancia hospitalaria; ${ }^{(6-7)}$ cuyas características son respeto, información, interés y amabilidad, entre otras.

A partir de la definición operacional, el indicador de trato de digno de enfermería se diseñó considerando 11 criterios, ${ }^{(6)}$ los cuales tienen su sustento en las condiciones señaladas en el Código de Ética de Enfermeras y Enfermeros en México, ${ }^{(11-13)}$ Carta de los Derechos Generales de las Pacientes y los Pacientes,(14) así como en el Artículo 51 de la Ley General de Salud, ${ }^{(15)}$ entre otros. Estos criterios son los siguientes: ${ }^{(10)}$

1. Porcentaje de pacientes a los cuales la enfermera (o) saluda en forma amable.

2. Porcentaje de pacientes a los que la enfermera (o) se presenta.

3. Porcentaje de pacientes a los que la enfermera(o) se dirige por su nombre.

4. Porcentaje de pacientes a los que la enfermera(o) le explica sobre los cuidados o actividades que le va realizar.

5. Porcentaje de pacientes que dentro de lo posible, su estancia fue agradable.

6. Porcentaje de pacientes a los que la enfermera(o) procura las condiciones necesarias que guardan su intimidad y/o pudor.

7. Porcentaje de pacientes a los que la enfermera(o) le hace sentirse segura (o) al atenderle.

8. Porcentaje de pacientes a los que la enfermera(o) lo trata con respecto.

9. Porcentaje de pacientes a los que la enfermera(o) le enseña al paciente o a su familiar de los cuidados que debe tener respecto a su padecimiento.

10. Porcentaje de pacientes con los que hay continuidad en los cuidados de enfermería a las 24 horas del día. 
11. Porcentaje de pacientes que se sienten satisfechos con el trato que le proporciona la enfermera (o).

Finalmente, el indicador de trato digno de enfermería se define como el porcentaje de pacientes atendidos por el personal de enfermería que respondieron afirmativamente a los 11 criterios o variables determinados para trato digno( ${ }^{(6)}$.

En base al cumplimiento de los criterios del indicador se definen estándares, que se representan mediante una semaforización de colores, donde verde significa calificación mínima esperada por el cumplimiento de los criterios establecidos para el indicador respectivo, amarillo indicador en riesgo, y rojo indicador que no cumple con los estándares de calidad de la atención en salud $^{(10)}$.

El estándar de calificación mínima esperada por el cumplimiento de los criterios establecidos para el indicador de trato digno de enfermería es de 95\%. La semaforización se asigna como verde para un estándar mayor a $95 \%$, amarillo entre 90 y 95\%, rojo cuando el estándar es menor a $90 \%$. El cálculo del estándar de calificación $(\mathrm{E})$, se realiza con la siguiente fórmula: ${ }^{(6)}$

\section{$E=B / C \times 100$}

Dónde:

$B=$ Total de pacientes o familiares encuestados, que fueron atendidos por el personal de enfermería, que respondieron afirmativamente a los 11 criterios determinados para trato digno en el servicio y período a evaluar.

$C=$ Total de pacientes encuestados en el mismo servicio y período.

Debido al impacto del trato digno en el desempeño de la profesión de enfermería, este trabajo tiene como propósito describir la percepción de la calidad de atención recibida por los usuarios de servicios de salud, mediante la revisión de los resultados del indicador de trato digno de enfermería en el segundo nivel de atención, durante el periodo de 2010-2015 en los diferentes establecimientos del Sistema Nacional de Salud, que participan en el sistema INDICAS, así como identificar los criterios de este indicador que puedan representar un mayor reto para el profesional de enfermería. Además, la información que se obtenga coadyuvará en una mejor comprensión de la evolución en la profesionalización de enfermería en México. 
FIGURA 1.

Indicador trato digno de Enfermería en el Sistema Nacional de Salud, 2010-2015.

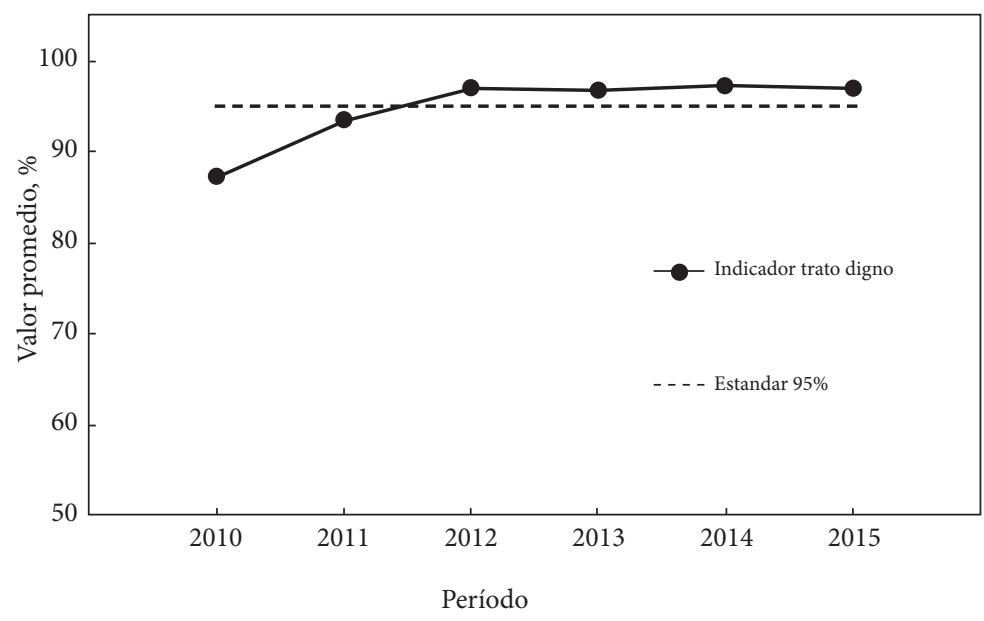

Fuente: http//dgces.salud.gob.mx/INDICASII

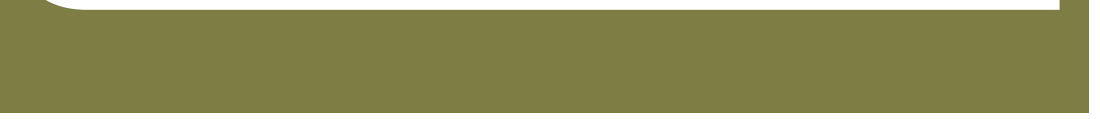

Figura 1

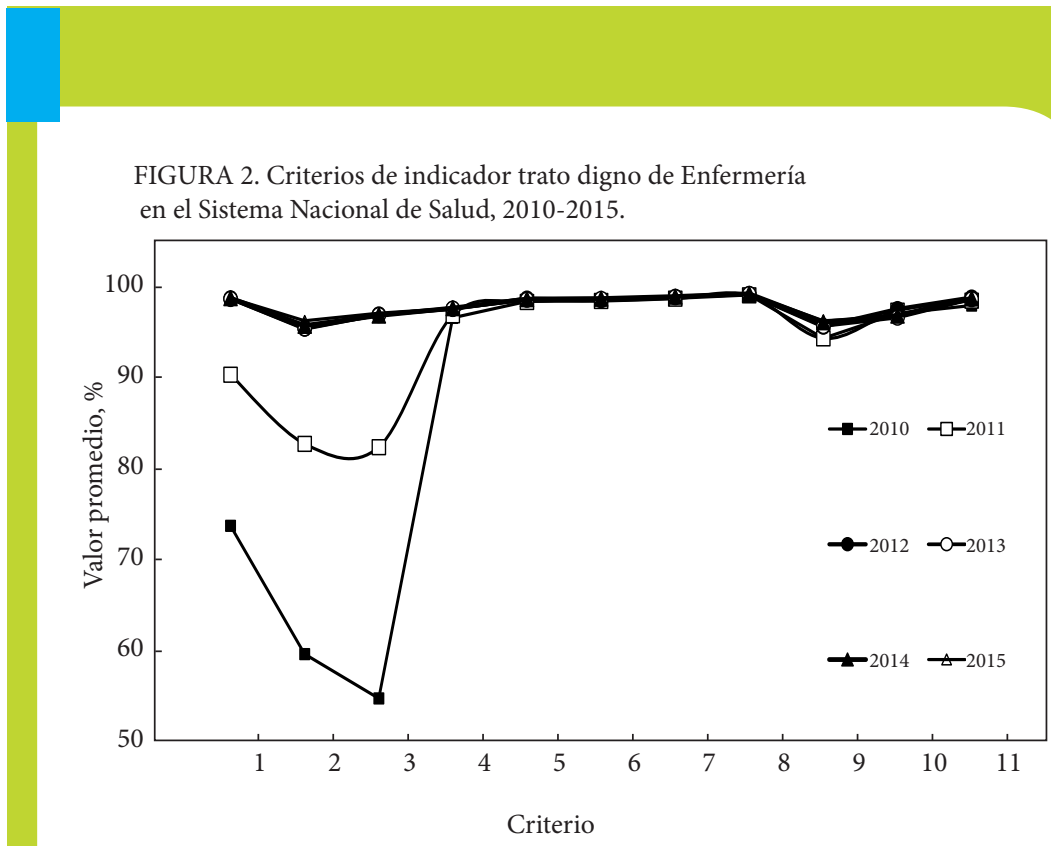

Fuente: http//dgces.salud.gob.mx/INDICASII

Figura 2.

\section{METODOLOGÍA}

La información analizada se obtuvo del portal de internet de la Dirección General de Calidad y Educación en Salud (DGCES), sección INDICAS. ${ }^{(10)}$ Los resultados que se presentan en este trabajo corresponden al promedio anual (tres cuatrimestres) de los porcentajes reportados en el sistema INDICAS para cada una de los 11 criterios definidos previamente para el indicador de trato digno de enfermería, así como el promedio de los porcentajes reportados para el indicador durante el período 2010-2015.

La información que presenta la DGCES a través de INDICAS procede de los establecimientos de atención médica públicos y privados del país inscritos en este sistema. En estos establecimientos se realiza la medición de indicadores de acuerdo a cédulas específicas, posteriormente se ingresa la información al sistema y se genera un reporte que se envía electrónicamente al sistema de información para indicadores de calidad (cada cuatro meses). Posteriormente, en el portal de INDICAS se presentan los resultados de las mediciones clasificados por índices, los cuales están constituidos por indicadores y éstos a su vez por criterios o variables.

\section{RESULTADOS}

\section{Indicador de trato digno de enfermería}

En la figura 1 se presenta el comportamiento del indicador de trato digno durante el período de 2010-2015. De acuerdo con esta figura, durante el año 2010 y 2011 no se cumplió con el estándar de calificación mínima esperada de 95\%, se encuentra en semáforo rojo. A partir de 2012 el promedio de calificación fue mayor de $95 \%$, por lo este indicador se encuentra en semáforo verde.

\section{Criterios de indicador de trato digno de enferme- ría}

Con el propósito de definir los criterios que impactan en cumplimiento del estándar para el indicador de trato digno de enfermería, en la figura 2 se presenta el comportamiento de cada uno de los criterios durante el período de 2010-2015.

De acuerdo con esta figura, durante el año 2010 y 2011 los criterios que no cumplieron con el estándar de calificación mínima esperada de 95\%, es decir, que se encuentran en semáforo rojo, son: 1) porcentaje de pacientes a los cuales la enfermera (o) saluda en forma amable, 2) porcentaje de pacientes a los que la enfermera (o) se presenta, y 3) porcentaje de pacientes a los que la enfermera(o) se dirige por su 
nombre. Además, en los 2 años la tendencia fue la misma; los criterios con menor valor fueron el 3, 2, y 1 respectivamente. Resulta importante destacar que aunque en el período 2010-2011 no se cumplió con el estándar, hubo una mejora significativa en estos tres criterios. También, se observa que el criterio 9: porcentaje de pacientes a los que la enfermera (o) le enseña al paciente o a su familiar de los cuidados que debe tener respecto a su padecimiento, se desplaza mínimamente del estándar (94\%), por lo que se encuentra en semáforo amarillo.

En congruencia con resultados de 2011 para el indicador de trato digno de enfermería en el Sistema Nacional de Indicadores de Calidad en Salud (INDICAS), se reportó por Nieto-González et al, (16) el incumplimiento de cada uno de los 11 criterios del indicador durante la atención proporcionada a la mujer embarazada que acudió a consulta prenatal en un hospital de segundo nivel en el Distrito Federal. Sobresaliendo en incumplimiento los criterios: el que las enfermeras no se presentan con el paciente, el no llamarlos por su nombre y no resguardar su intimidad y pudor.

A partir de 2012, se cumple con el estándar $\geq 95 \%$ para cada uno de los 11 criterios del indicador de trato digno de enfermería en los establecimientos de salud inscritos en INDICAS.

Sin embargo, existen estudios donde se indica incumplimiento del indicador de trato digno de enfermería. En 2012, García-Gutiérrez y Cortés-Escarcéga (13) reportaron el incumplimiento del indicador en un hospital de segundo nivel del Estado de México, el cual es mayormente afectado porque la enfermera no se presenta con el paciente, no le proporciona una explicación previa a los procedimientos que le realiza, no favorece las condiciones necesarias que resguardaran su intimidad y/o pudor durante su atención, y no proporciona información al paciente y/o familiar sobre su padecimiento. Resulta importante mencionar que en este estudio, también se indica que un alto porcentaje de satisfacción con el trato que proporciona el profesional de enfermería, está relacionado con los resultados obtenidos del proceso de atención. Esta información es sugestiva, de que la percepción del paciente o familiar está determinada por múltiples factores inherentes al proceso de atención, así como a las características del propio paciente, y tiende a percibir un trato de enfermería adecuado cuando ocurre una mejora en el estado de salud.

\section{DISCUSIÓN}

Los resultados obtenidos, indican que hubo una mejora continua y sostenida en el indicador de trato digno de enfermería durante el periodo de 2010 a 2015, hasta que a partir de 2012 se cumplió con el estándar definido para este indicador, probablemente porque a partir de la medición fue posible identificar las áreas de oportunidad y posteriormente, implementar estrategias conjuntas entre los profesionales de enfermería.

Por otro lado, tres criterios son los que han representado mayor área de oportunidad, estos son: 1) porcentaje de pacientes a los cuales la enfermera (o) saluda en forma amable, 2) porcentaje de pacientes a los que la enfermera (o) se presenta y 3 ) porcentaje de pacientes a los que la enfermera (o) se dirige por su nombre.

Los criterios 1 y 2, probablemente están relacionados con el ambiente laboral donde se desempeña el profesional de enfermería, así como a características personales, profesionales y motivacionales. Sin embargo, el criterio 3, está estrictamente relacionado con el principio de respeto a la individualidad del paciente, es decir con el aspecto ético de la profesión. Por lo que este criterio, invita a la reflexión de que los pacientes son ante todo personas en una situación vulnerable y por tanto no pueden ser convertidos a números, camas o aparatos. Es requisito dirigirse a los pacientes por su nombre, además es un acto que les genera satisfacción, porque probablemente lo traducen a interés, empatía, motivación, agrado por el trabajo, etc.

Otra variable que ha representado riesgo, es: 9) porcentaje de pacientes a los que la enfermera (o) le enseña al paciente o a su familiar de los cuidados que debe tener respecto a su padecimiento. Este criterio, se puede relacionar específicamente con la función docente de enfermería, y la promoción del autocuidado.

Actualmente, se cumple con el estándar para el indicador de trato digno de enfermería, hoy por hoy el cuidado humanizado es un atributo de la profesión de enfermería. Sin embargo, existe el riesgo latente de la modernización que lleva consigo tecnología que confunde, por tanto, se requiere realizar una medición objetiva y continua de nuestros procesos, para adaptar y transformar de acuerdo a las necesidades y satisfacción de los pacientes, concibiéndolos en todo momento como una unidad biopsicosocial y espiritual. 
Resulta importante mencionar, que probablemente entre los pacientes más vulnerables al incumplimiento del indicador de trato digno, están los pacientes pediátricos, ${ }^{(17)}$ así como los que se encuentran en unidades de cuidados intensivos (UCI). Se ha reportado, que entre los factores estresantes para los pacientes ingresados en la UCI están el no tener privacidad, no saber cuándo las cosas serán hechas, no recibir explicación sobre el tratamiento, y que el profesional de enfermería no se presente por su nombre. ${ }^{(18)}$

Finalmente, la mejora gradual y sostenida para el indicador de trato digno de enfermería en el Sistema Nacional de Salud, se puede considerar como evidencia del proceso de profesionalización de enfermería en México. ${ }^{(19-21)}$

Es necesario continuar el desarrollo sostenido de una cultura de calidad en la profesión de enfermería desde los ámbitos laborales hasta los académicos. Los profesionales insertos en el mercado laboral deberán ser corresponsables de diseñar e implementar las estrategias que les permitan actualizar y aplicar los conocimientos científicos, técnicos, éticos y humanísticos en el cuidado enfermero. Las autoridades de enfermería, deberán tener el compromiso de realizar las gestiones necesarias que coadyuven en la calidad de atención, así como de la inclusión participativa de personal operativo en estrategias de calidad.

En el ámbito académico, se deberá promover y fortalecer una formación integral del estudiante, con apego a una cultura de calidad que responda a las necesidades y políticas de salud del país.

\section{CONCLUSIONES}

La aplicación del enfoque de calidad en la atención de enfermería implica un componente ético y humanístico sostenido como antesala de cualquier proceso, donde la relación paciente-profesional de enfermería sea de respeto y compromiso del uno por el otro. Se debe estar consciente que la calidad es en gran parte percepción, por tanto, tiene ese componente subjetivo que está en función de las características y expectativas del paciente. Además, el trato humanizado no incrementa el trabajo del profesional de enfermería, pero si coadyuva en la atención de calidad, y favorece el reconocimiento, identidad y estatus social de la profesión.

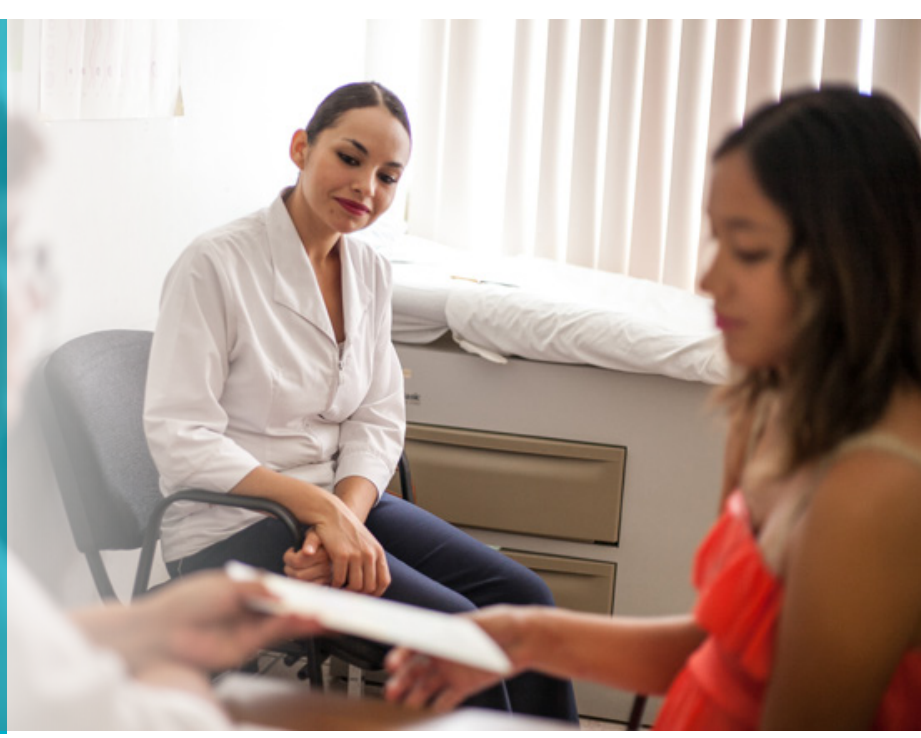


1. Salinas P. Hugo, et al. Indicadores de calidad de asistencia en obstetricia. Rev Chil Obstet Ginecol 2006; 71(2):114-20.

2. Donabedian A. Continuidad y cambio en la búsqueda de la calidad. Conferencia magistral. Salud pública en México. 1993; 35(3): 238-47.

3. Donabedian A. Reflexiones sobre calidad asistencial. Revista Calidad Asistencial. 2001; 16.

4. Secretaría de Salud. Programa de acción específico. Estrategia nacional para la consolidación de la calidad en los establecimientos y servicios de atención médica. Programa sectorial de salud 2013-2018. México, D.F. 2013.

5. Alvarado-Gallegos E, et al. Evaluación Participativa de los Indicadores de Calidad de Enfermería. Desarrollo Cientif Enferm 2011; 1(6): 212-18.

6. Comisión Interinstitucional de Enfermería, Secretaría de Salud Evaluación de la calidad de los servicios de enfermería. Tres indicadores de aplicación hospitalaria. Secretaría de Salud. México, D.F. 2003

7. Nava Galán M, Zamora Ruiz P. Percepción del trato digno de los familiares de los enfermos neurológicos en el Instituto Nacional de Neurología y Neurocirugía. Enf Neurol (Mex) 2010; 9(1): 328.

8. Hernández-Méndes C, Azcona-Cruz, M. Calidad del cuidado de enfermería en el Hospital Regional "Presidente Juárez" del ISSSTE, Oaxaca. Avances en Ciencia, Salud y Medicina 2013; 1(3): 95-9.

9. Ceballos Vásquez, P. Desde los ámbitos de enfermería, analizando el cuidado humanizado. Ciencia y Enfermería 2010; XVI (1): 31-5.

10. Secretaria de Salud. INDICAS. Sistema Nacional de Indicadores en Salud. Dirección General de Calidad y Educación en Salud. Indicadores de calidad por enfermería. Disponible en: http:// dgces.salud.gob.mx

11. Troncoso M, Valenzuela S. Cuidado humanizado: un desafío para las enfermeras en los servicios hospitalarios. Acta Paul Enferm 2007; 20(4) 499-503.

12. Carrillo $\mathrm{M}$, et al. Cumplimiento del indicador de calidad trato digno proporcionado por el personal de enfermería. Rev Enferm Inst Mex Seguro Soc 2009; 17 (3): 139-42.

13. García-Gutiérrez C, Cortés-Escarcéga, I. Percepción del usuario del trato digno por enfermería en un hospital del Estado de México. Rev CONAMED 2012; 17(1):18-23.

14. Secretaría de salud, CONAMED. Carta de los derechos generales de las pacientes y los pacientes. 2001.

15. Cámara de Diputados del H. Congreso de la Unión. Ley general de Salud. Nueva Ley publicada en el Diario Oficial de la Federación el 7 de febrero de 1984. Últimas reformas publicadas DOF 27-04-2010. México, D.F.

16. Nieto-González $\mathrm{L}$, et al. Percepción del trato digno por la mujer embarazada en la atención obstétrica en enfermería. Rev CONAMED 2011; 16(1):5-1.

17. Rodríguez Ramos M, Velázquez Quintero, A. Trato digno en los cuidados de enfermería

18. al paciente pediátrico. Revista Mexicana de Enfermería Cardiológica 2014; 22 (3): 96-7.

19. Fernandes da Cruz Silva L, et al. Estrés del paciente en UCI: visión de los pacientes y del equipo de enfermería. Enfermería global 2013; 32: 88-103.

20. Orozco Reyes $\mathrm{O}$, et al. Factores asociados a la profesionalización de Enfermería en México. Rev CONAMED 2015; 207(4):41-7.

21. Rubio Domínguez, S. Profesionalización de Enfermería en México. Revista Mexicana de Enfermería Cardiológica 2010; 18 (1): 4-6.

22. González Carrillo E, et al. La profesionalización en enfermería: hacia una estrategia de cambio. Tecnociencia Chihuahua. 2012;6(1): 1-8.

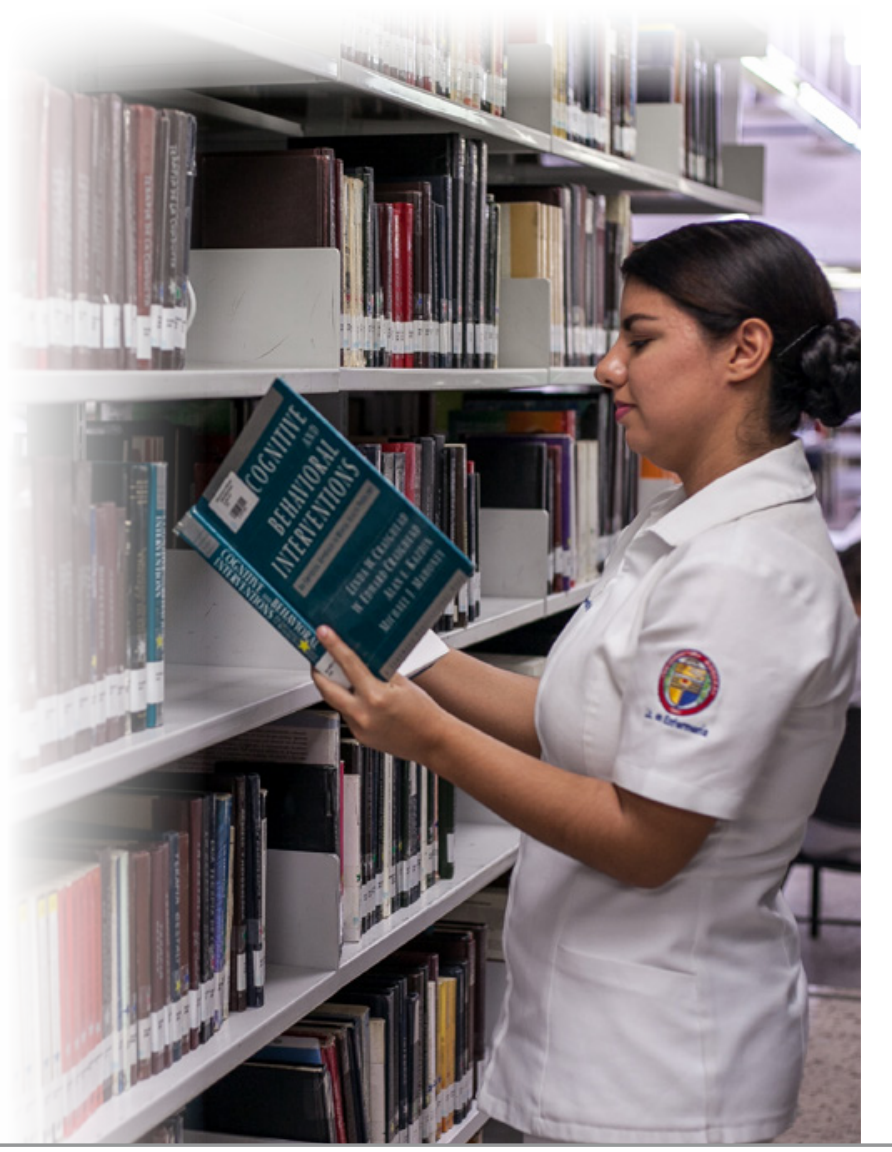

